\title{
Clinical efficacy of epicardial application of drug-releasing hydrogels to prevent postoperative atrial fibrillation
}

\author{
William Wang, MD, PhD, ${ }^{\mathrm{a}}$ Yun Qing Mei, MD, PhD, ${ }^{\mathrm{b}}$ Xin Hiu Yuan, MD, ${ }^{\mathrm{c}}$ and Xiao Dong Feng, $\mathrm{MD}^{\mathrm{d}}$
}

\begin{abstract}
Objective: Postoperative atrial fibrillation is the most frequent complication arising after cardiac surgery, occurring in $40 \%$ of cases. The treatment of postoperative atrial fibrillation with epicardial amiodarone/corticosteroid hydrogel delivery can increase efficacy and reduce side effects. To further evaluate whether amiodarone hydrogel is superior to corticosteroid hydrogel or placebo, we performed a randomized prospective study in 150 patients with coronary artery bypass grafting to compare the effectiveness with different epicardial drug approaches in the postoperative period.
\end{abstract}

Methods: After institutional review board approval, 150 patients, from January 2012 to July 2014, who had undergone cardiac surgery were randomized to 3 equal groups. Group I received poly-based hydrogel with amiodarone, and group II received poly-based hydrogel with triamcinolone. Both hydrogels were sprayed diffusely over the biatrial epicardium. The control group underwent the procedure with only hydrogel spray. Continuous telemetry monitored for postoperative atrial fibrillation, and amiodarone or triamcinolone levels in the atria, plasma, and tissue were measured postoperatively. Daily electrocardiographic parameters were measured until postoperative day 14.

Results: The incidence of postoperative atrial fibrillation was significantly less in group I, with 4 of 50 patients ( $8 \%$ ) incurring atrial fibrillation compared with 11 of 50 patients $(22 \%)$ in group II and 13 of 50 patients $(26 \%)$ in the control group $(P<.01)$. The mean amiodarone and triamcinolone concentrations in the atria $(12.06 \pm 3.1 / 1.5 \pm 0.7)$ were significantly greater than those in the extracardiac tissues $(1.32 \pm 0.9 / 0.2 \pm 0.4 ; P<.01)$. The plasma amiodarone and triamcinolone levels remained below the detection limit $(<8 \mu \mathrm{g} / \mathrm{mL}$ and $<0.2 \mu \mathrm{g} / \mathrm{mL}$ ) during the 14 days of follow-up. Bradycardia was observed less in the control group $(93 \pm 18)$ than in study group I $(76 \pm 29 ; P<.01)$.

Conclusions: Epicardial application of amiodarone-releasing adhesive hydrogel is a less-invasive, well-tolerated, quick, and effective therapeutic option for preventing postoperative atrial fibrillation with minimal risk of extracardiac adverse side effects. However, there was no clinical evidence that epicardial corticosteroid prevented postoperative atrial fibrillation. (J Thorac Cardiovasc Surg 2016;151:80-5)

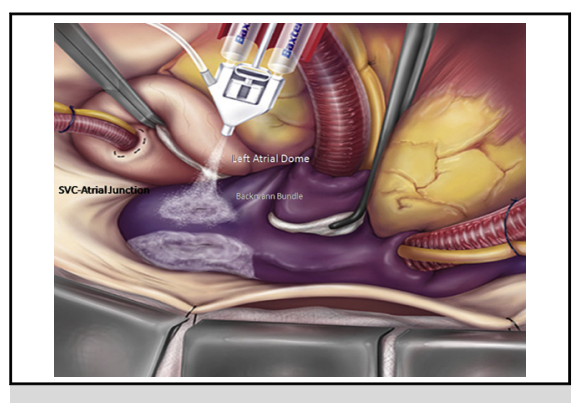

Epicardial application of drug-releasing adhesive hydrogel.

\section{Central Message}

Epicardial application of drug-releasing adhesive hydrogel is an effective therapeutic option for preventing postoperative $\mathrm{AF}$.

\section{Perspective}

Epicardial application of amiodarone-releasing adhesive hydrogel is a less-invasive, welltolerated, quick, and effective therapeutic option for preventing POAF with minimal risk of extracardiac adverse side effects. However, there was no clinical evidence that epicardial corticosteroid prevented POAF.

See Editorial Commentary page 86 .
From the ${ }^{a}$ Department of Cardiovascular Surgery, Scripps Memorial Hospital, La Jolla, Calif; 'bepartment of Cardiovascular Surgery, Shanghai Tongji Hospital, Shanghi, China; 'Department of Cardiovascular Surgery, Changzhi Peace Hospital, Changzhi, Shanxi, China; ${ }^{\mathrm{d}}$ Department of Cardiovascular Surgery, Shanghai East Hospital, Shanghai, China.

Read at the 95th Annual Meeting of The American Association for Thoracic Surgery, Seattle, Washington, April 25-29, 2015.

Received for publication April 21, 2015; revisions received June 5, 2015; accepted for publication June 16, 2015; available ahead of print Aug 5, 2015

Address for reprints: Xiao Dong Feng, MD, 150 Jimo Rd, Pudong, Shanghai, China 200135 (E-mail: fxd9188zw@hotmail.com).

$0022-5223 / \$ 36.00$

Copyright (c) 2016 by The American Association for Thoracic Surgery

http://dx.doi.org/10.1016/j.jtcvs.2015.06.061
Postoperative atrial fibrillation (POAF) is the most frequent complication arising after cardiac surgery, occurring in $44 \%$ of cases. ${ }^{1,2}$ The pathophysiologic mechanism of POAF remains unknown. It increases patient mortality and morbidity, hospitalization duration, and healthcare resources. Currently, the available conventional therapies for preventing POAF are suboptimal. Amiodarone, a class III antiarrhythmic medicine, is the most effective drug for prophylaxis and treatment. Clinical studies have demonstrated the efficacy of oral and intravenous amiodarone therapy to prevent POAF. Despite its superior 


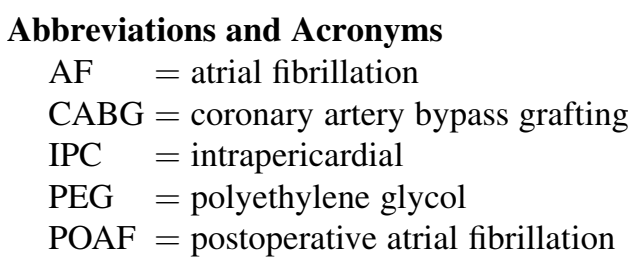

efficacy, amiodarone therapy has several disadvantages, including requirement of a loading period, interactions with many other drugs, and, most important, serious extracardiac side effects, such as thyroid dysfunction, pulmonary toxicity, and hepatic toxicity. ${ }^{3,4}$

Considerable experimental and clinical evidence suggest that POAF is multifactorial. Facilitating factors can be classified as acute factors caused by the surgical intervention (inflammation) and chronic factors related to structural heart disease. Cardiac surgery, especially when accompanied by cardiopulmonary bypass, causes a systemic inflammatory response that may be in part responsible for POAF. Complement, C-reactive protein complex levels, and white blood cell count-markers of an inflammatory reaction-are increased in patients after cardiac surgery. Elevated C-reactive protein complex and C-reactive protein complex-complement levels after cardiac surgery are predictive of POAF. ${ }^{5}$ The utility of anti-inflammatory agents for preventing POAF also has been studied in experimental animals and clinically. Prednisone given orally prevents inducible atrial flutter in a canine sterile pericarditis model. ${ }^{6}$ A similar study showed that corticosteroids decreased the frequency and duration of atrial fibrillation (AF) in an animal model of cardiac surgery. ${ }^{7}$ Two prospective, randomized clinical trials of perioperative corticosteroids showed a significant reduction in POAF. ${ }^{8}$

The concept of local amiodarone/corticosteroid delivery to the myocardium has been investigated in several animal studies by intrapericardial (IPC) infusion of amiodarone/steroid solutions. ${ }^{9,10}$ This approach is not applicable in the postoperative patient because the pericardium is usually left open and in communication with a mediastinal drain. Along with bleeding and effusions, any drug solution instilled at the time of surgery would be rapidly evacuated. A few studies researching the effectiveness of the topical application of a biodegradable disc with amiodarone or amiodarone-eluting bilayered patch have reported encouraging results. ${ }^{11,12}$ However, the amount of exposure to the atrial epicardial surface is often limited using those applications. Biatrial epicardial application of drugreleasing hydrogel might offer the advantage of a more localized (biatrial targeted) drug delivery.

Surgical sealant CoSeal (Baxter Healthcare, Fremont, Calif) consists of 2 biodegradable synthetic polyethylene glycol (PEG) polymers that are mixed at the time of application, forming a strong hydrogel that vigorously adheres to tissue. ${ }^{13,14}$ These properties make this biomatrix attractive as a vehicle for local drug delivery. ${ }^{15} \mathrm{We}$ reasoned that addition of amiodarone/steroid to this hydrogel would produce a drug-releasing matrix capable of suppressing atrial tachyarrhythmias while systemic drug levels remain low. The purpose of this prospective study is to evaluate the efficacy of amiodarone versus corticosteroid-loaded hydrogels applied to the atria to prevent POAF.

\section{MATERIAL AND METHODS}

After institutional review board and ethical research committee approval, 150 patients undergoing conventional coronary artery bypass grafting $(\mathrm{CABG})$ were randomized to 3 equal groups after obtaining informed consent. Group I received poly-based hydrogel with amiodarone, and group II received poly-based hydrogel with triamcinolone. Both hydrogels were sprayed diffusely over the biatrial epicardium. The control group underwent the procedure with the hydrogels spray only. Patients were excluded if they had a history of pacemaker/automatic internal cardiac defibrillator or significant preoperative events developed, such as cardiogenic shock or ventricular tachyarrhythmias (Table 1).

CoSeal Surgical Sealant, a sprayable polymeric matrix, was obtained via Baxter Healthcare. The kit is composed of 2 synthetic PEGs, a dilute hydrogen chloride solution, and a sodium phosphate/sodium carbonate solution. Amiodarone hydrochloride powder (Sigma-Aldrich, St Louis, Mo) $1 \mathrm{mg} / \mathrm{kg}$ bodyweight or triamcinolone acetonide (Sandoz Inc, Princeton, NJ) $0.5 \mathrm{mg} / \mathrm{kg}$ was first added to the PEG powder and then mixed with solutions until the drug was completely dissolved. All patients underwent dissection of the superior vena cava-atrial junction area and the free left atrial anterior wall, which was adjacent to the transverse sinus (Bachmann's bundle area). Temporary epicardial atrial and ventricular pacing wires were inserted in all patients at the end of surgery. Before the sternum was closed, hydrogels were sprayed diffusely in a thin layer onto the exposed epicardial surfaces with a $\mathrm{Co}_{2}$ driver set over the right atrial lateral wall, left atrial appendage, and transverse sinus area (Figure 1). The pericardium was approximated with interrupted suture, and a single mediasternal chest tube was placed retromediastinal above the pericardium. The myocardial venous blood sampling was obtained from the coronary venous sinus under fluoroscopic control during postoperative day 3 , right atrial endomyocardial biopsy was performed from areas adjacent to the fossa ovalis for measurements of myocardial amiodarone/triamcinolone concentration, and abdominal extraperitoneal adipose tissue within the chest tube site was biopsied during chest tube removal on postoperative day 3. Amiodarone/triamcinolone plasma concentrations were also measured in the blood drawn from a peripheral vein during postoperative days 2 and 5. The amiodarone and triamcinolone assays were performed by the standard method of high-performance liquid chromatography (HP-Series 1090, Hewlett Packard, Palo Alto, Calif). A cardiac enzyme panel was measured on postoperative days 1, 3, and 5, and before discharge to monitor the local atrial myocardial injury. Continuous telemetry was monitored for POAF. Daily complete blood count and electrocardiography parameters (RR, PQ, QT, and maximal Tpeak-Tend intervals, and $\mathrm{P}$ and $\mathrm{QRS}$ widths) were measured until postoperative day 14. After the operation, the patient in the control group received intravenous or oral amiodarone for new-onset AF. Electrical cardioversion was performed before hospital discharge in any patient who was not in normal sinus rhythm.

All values are expressed as the mean \pm standard deviation. Statistical analysis comparing the data between the 2 groups was performed with the chi-square test for categoric variables. Continuous variables were compared by means of 2-tailed Student $t$ tests and Kruskal-Wallis test 
TABLE 1. Patient demographic characteristics

\begin{tabular}{|c|c|c|c|c|c|}
\hline Characteristic & Group I & Group II & Control group & Total value & Univariate $P$ value \\
\hline Patient (N) & 50 & 50 & 50 & 150 & NS \\
\hline Median age, $\mathrm{y}$ & $53 \pm 7.4$ & $52 \pm 9.1$ & $51 \pm 7.9$ & - & NS \\
\hline $\mathrm{M} / \mathrm{F}$ & $30 \pm 20$ & $29 / 21$ & $31 / 19$ & - & NS \\
\hline Prior MI & $12(24 \%)$ & $14(28 \%)$ & $13(26 \%)$ & 39 & NS \\
\hline $\mathrm{CHF}$ & $13(26 \%)$ & $9(18 \%)$ & $12(24 \%)$ & 44 & NS \\
\hline Preoperative IABP & $6(12 \%)$ & $3(6 \%)$ & $4(8 \%)$ & 13 & NS \\
\hline Prior CABG & $2(4 \%)$ & $4(8 \%)$ & $6(12 \%)$ & 12 & NS \\
\hline Prior PCI & $31(62 \%)$ & $27(54 \%)$ & $24(48 \%)$ & 82 & NS \\
\hline Hypertension & $14(28 \%)$ & $16(32 \%)$ & $11(22 \%)$ & 41 & NS \\
\hline Diabetes & $15(30 \%)$ & $17(34 \%)$ & $19(38 \%)$ & 51 & NS \\
\hline Chronic lung disease & $19(38 \%)$ & $22(44 \%)$ & $23(46 \%)$ & 45 & NS \\
\hline PVD & $9(18 \%)$ & $6(12 \%)$ & $7(14 \%)$ & 54 & NS \\
\hline Prior stroke & $6(12 \%)$ & $5(10 \%)$ & $8(16 \%)$ & 19 & NS \\
\hline Smoking (last 6 mo) & $37(74 \%)$ & $35(70 \%)$ & $39(78 \%)$ & 111 & NS \\
\hline Body mass index $>30$ & $24(48 \%)$ & $21(42 \%)$ & $19(38 \%)$ & 64 & NS \\
\hline$\beta$-blockers & $16(32 \%)$ & $11(22 \%)$ & $14(28 \%)$ & 31 & NS \\
\hline Calcium channel blockers & $4(8 \%)$ & $5(10 \%)$ & $7(14 \%)$ & 16 & NS \\
\hline Aspirin & $40(80 \%)$ & $31(62 \%)$ & $37(74 \%)$ & 108 & NS \\
\hline
\end{tabular}

$M / F$, Male/female; $M I$, myocardial infarction; $I A B P$, intra-aortic balloon pump; $C A B G$, coronary artery bypass grafting; $P C I$, percutaneous coronary intervention; $P V D$, peripheral vascular disease.

when appropriate. Data collected were analyzed using the Number Cruncher Statistical Systems software (NCSS, Kaysville, Utah).

\section{RESULTS}

There were no epicardial amiodarone/triamcinolone hydrogel-related complications. The highest incidence of troponin $\mathrm{T}$ detection was observed in 2 patients $(1.3 \%)$ in group I and 3 patients ( $2 \%)$ in group II with preoperative acute myocardial infarction. Transient bradycardia that required temporary pacing was seen in 7 patients of group I. Overall hospital mortality was $2 \%$. Stroke occurred in 3 patients $(2 \%)$, and 7 patients underwent reoperation for bleeding $(4.6 \%)$ (Table 2). Mean follow-up time was $8 \pm 7$ months (range, 6-17 months). The incidence of

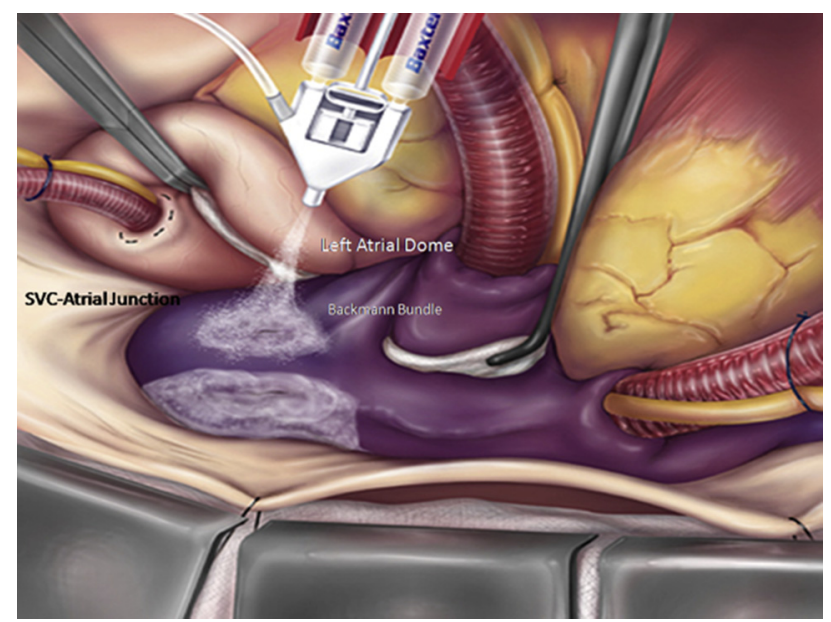

FIGURE 1. Biatrial epicardial application of drug-releasing hydrogel. $S V C$, Superior vena cava.
POAF was significantly less in group I with 4 of 50 patients $(8 \%)$ incurring AF compared with 11 of 50 patients $(22 \%)$ in group II and 13 of 50 patients (26\%) in the control group $(P<.01)$. In group I, the coronary venous sinus blood concentration of amiodarone $(10.09 \pm 3.6 \mu \mathrm{g} / \mathrm{mL})$ was far higher than in the peripheral plasma $(1.43 \pm 1.6 \mu \mathrm{g} / \mathrm{mL})$ $(P<.01)$. Plasma concentrations of both amiodarone and desethylamiodarone remained below detection limits $(<8 \mu \mathrm{g} / \mathrm{mL})$ during 7 days of follow-up. The coronary venous sinus blood concentration of triamcinolone $(1.7 \pm 0.2 \mu \mathrm{g} / \mathrm{mL})$ in group II was higher than in the peripheral plasma $(0.9 \pm 0.4 \mu \mathrm{g} / \mathrm{mL})$ during postoperative day 1 to 3 , and plasma concentrations of triamcinolone remained below detection limits $(<0.2 \mu \mathrm{g} / \mathrm{mL})$ during 4 to 7 days of follow-up. The mean amiodarone and triamcinolone concentrations in the atria $(12.06 \pm 3.1 \mu \mathrm{g} / \mathrm{g}$ and

TABLE 2. Postoperative complications

\begin{tabular}{llccc}
\hline \multicolumn{1}{c}{ Complications } & Group I & Group II & Control group & Total value \\
\hline Patient $(\mathrm{N})$ & 50 & 50 & 50 & 150 \\
Bradycardia & $7(14 \%)$ & $1(2 \%)$ & $0(0 \%)$ & $8(5.3 \%)$ \\
POAF & $4(8 \%)$ & $11(22 \%)$ & $13(26 \%)$ & $28(19 \%)$ \\
Stroke & $0(0 \%)$ & $2(4 \%)$ & $1(2 \%)$ & $3(2 \%)$ \\
bleeding & $2(4 \%)$ & $4(8 \%)$ & $1(2 \%)$ & $7(5 \%)$ \\
Respiratory failure & $6(12 \%)$ & $4(8 \%)$ & $9(18 \%)$ & $19(12 \%)$ \\
Pneumonia & $2(4 \%)$ & $1(2 \%)$ & $0(0 \%)$ & $3(2 \%)$ \\
Plural effusion & $5(10 \%)$ & $3(6 \%)$ & $2(4 \%)$ & $10(6 \%)$ \\
Renal failure & $3(6 \%)$ & $6(12 \%)$ & $1(2 \%)$ & $10(6 \%)$ \\
Sternal infection & $0(0 \%)$ & $1(2 \%)$ & $1(2 \%)$ & $2(1.3 \%)$ \\
Leg wound infection & $3(6 \%)$ & $2(4 \%)$ & $4(8 \%)$ & $9(6 \%)$ \\
Delirium & $4(8 \%)$ & $2(4 \%)$ & $1(2 \%)$ & $7(5 \%)$ \\
\hline
\end{tabular}

POAF, Postoperative atrial fibrillation. 
$1.5 \pm 0.7 \mu \mathrm{g} / \mathrm{g}$ ) were significantly greater than in the extracardiac tissues $(1.32 \pm 0.9 \mu \mathrm{g} / \mathrm{g}$ and $0.2 \pm 0.4 \mu \mathrm{g} / \mathrm{g})$ $(P<.01)$. The mean heart rate averaged from $76 \pm 29$ beats/min within 7-day periods in group I compared with $93 \pm 18$ beats/min and $86 \pm 11$ beats/min in group II and the control group $(P<.01)$, QT intervals increased from $401 \pm 44 \mathrm{~ms}$ before amiodarone hydrogels to $412 \pm 53 \mathrm{~ms}$ after amiodarone therapy, and rate-corrected QT intervals (QTc) increased from $452 \pm 43 \mathrm{~ms}$ to $463 \pm 37 \mathrm{~ms}(P=.06)$ in group $\mathrm{I}$.

\section{DISCUSSION}

The main finding of the present study was that biatrial epicardial application of amiodarone-releasing hydrogel was effective in preventing and lowering the incidence of POAF; however, there was no clinical evidence that epicardial corticosteroid prevented POAF. The epicardial treatment was safe and well tolerated, and yielded persistently higher drug concentrations in the atria compared with the extracardiac tissues. The treatment was easy to apply and displays both biodegradability and immediate tissue adherence, allowing more localized drug delivery.

$\mathrm{AF}$ is a common and undesirable event after cardiac operation and is an important source of patient morbidity and increased resource use. The incidence of AF has been reported to be $27.6 \%$ after CABG, $48.8 \%$ after mitral valve replacement, $32.9 \%$ after aortic valve replacement, and higher after combined procedures. ${ }^{16}$ POAF is associated with an increased hospital stay and early stroke risk, and a reduction in-hospital and long-term survival. ${ }^{17}$ Although promising nonpharmacologic therapies, particularly ablation procedures, have been developed, pharmacotherapy still remains the cornerstone of $\mathrm{AF}$ treatment. At the present time, the most frequently applied drug worldwide for maintaining sinus rhythm in patients with $\mathrm{AF}$ is amiodarone, a highly lipophilic, multi-ion channel blocker. ${ }^{18}$ In comparison with other contemporary clinically used antiarrhythmic agents, it combines high effectiveness with a low risk of Torsade de pointes. However, major drawbacks of amiodarone involve its multiple systemic side effects, including thyroid, pulmonary, hepatic, and dermatologic toxicity, as well as its complex and inconvenient pharmacokinetic profile, including slow and extensive tissue accumulation and long half-life. To improve the effectiveness of drugs and minimize their systemic toxicity, 3 alternative applications for epicardial amiodarone delivery have been developed: IPC-infused amiodarone solutions, epicardially sprayed in situ amiodarone releasing adhesive hydrogels, and epicardially sutured amiodarone-eluting bilayered patches or disc. ${ }^{19-21}$ A number of studies have investigated local drug delivery of antiarrhythmic agents, including lidocaine, digoxin, amiodarone, beta-blockers, procainamide, ibutilide, sotalol, and flecainide. ${ }^{17,21,22}$ Pharmacokinetic advantages and significant effects on atrial electrophysiologic parameters were invariably demonstrated. IPC amiodarone infusion is ideally suited for patients undergoing noncardiac surgery with an intact pericardial sac because it provides a natural drug reservoir and space for epicardial drug delivery. The epicardial amiodarone-eluting bilayered patches or discs allow a more localized release of drugs, minimize drug leakage to the pericardial fluid to prevent disruption of ventricular electrophysiology, and produce long-term sustained drug delivery by biodegradable matrix. Nevertheless, epicardial patch application was considered somewhat difficult and time-consuming, and has limited the acceptance of left atrial wall because of the anatomic complexity of the left atrium. Epicardially sprayed in situ PEG-based amiodarone-releasing adhesive hydrogels that are currently used in this study are biodegradable amiodarone-loaded matrix adhesive to the epicardium and able to achieve sustained therapeutic myocardial concentrations. We have found the application to be technically simple and quick to accomplish, and most important, the hydrogel diffusingly covered both atrial surfaces without adding any complications. The thin atria seem to be suitable for this delivery method because of the high surface area/wall thickness ratio.

This study first clinically demonstrated that biatrial epicardial application of amiodarone-releasing hydrogel significantly reduced the incidence of POAF compared with the control group. It specifically increases the amiodarone concentration of the atrial tissue and thus becomes locally effective for the atrium. The atrial concentration of amiodarone was higher than in the other organs, and the blood concentration of amiodarone was below detectable levels. This was due to local high amiodarone concentration to the intracardial diffusion. Bolderman and colleagues ${ }^{12}$ reported that application of amiodarone-releasing hydrogel to the atrium produced 10-fold higher epicardial drug concentrations than endocardial concentrations in an animal study. The present study also found this delivery system minimizes drug release into the pericardial fluid, does not seem to disturb ventricular electrophysiology, and enables longer-lasting site-specific drug delivery. There was no QT/QTc interval prolongation, Torsade de pointes, or significant clinical bradycardia.

Considerable clinical evidence has demonstrated that intravenous corticosteroid significantly reduced the relative risk of POAF by $37 \%$ to $40 \%$ compared with placebo in patients undergoing CABG. In addition, several meta-analyses in which all randomized controlled trials were included $^{23}$ confirmed the beneficial effect of corticosteroid treatment over placebo and found systemic administration of hydrocortisone therapy feasible and well 
tolerated, and noted some serious complications associated with intravenous administration of the drug. The mechanisms by which corticosteroids prevent POAF are not entirely clear. The concentration of complement C-reactive protein complex, the number of white blood cells, and the concentration of inflammatory cytokines, all markers of increased inflammatory reaction concentration, are higher in patients with POAF than in patients who remain in sinus rhythm. ${ }^{24}$ Corticosteroids can target the inflammatory process and reduce exaggerated inflammatory reaction for the prevention of POAF in patients undergoing cardiac surgery; however, there is no consensus on which steroid, dose, or duration has the greatest benefit on anti-inflammatory activity. Recent investigations have indicated that cytokines play a key role in the inflammatory cascade associated with CPB. The levels of tumor necrosis factor- $\alpha$, interleukin-6, and interleukin- 8 were correlated with the duration of cardiac ischemia, and the myocardium is a major source of these cytokines during $\mathrm{CPB}$.

Increased risk of wound infections and gastrointestinal bleeding (stress ulcer), and hyperglycemia requiring insulin can be concerns with corticosteroid systemic therapy, and recent studies showed that IPC delivery of corticosteroid results in the most effective treatment of recurrent pericardial effusion. ${ }^{21}$ It improved the effectiveness of drugs and minimized their systemic toxicity. Epicardial delivery of anti-inflammatory corticosteroid adhesive hydrogels results in a higher ratio of myocardial-tosystemic drug concentrations, lowering the potential for side effects. Yoo and colleagues ${ }^{25}$ reported that a mixture of fibrin glue and corticosteroid was sprayed onto the epicardium during cardiac surgery in an animal model of POAF. The treatment dramatically reduced the burden of spontaneous postoperative atrial tachycardias compared with control animals. However, this study demonstrated that there were no significant differences in the occurrence of $\mathrm{AF}$ in group II with corticosteroid hydrogels $(22 \%$ in group II vs $26 \%$ in the control group), even if the mean triamcinolone concentrations in the atria were significantly greater than those in the extracardiac tissues. There are several possible explanations for these disparate findings. (1) Specific dosing of corticosteroids that may confer optimal protection against POAF is unknown. Converting the triamcinolone dosing to dexamethasone equivalence on the basis of total cumulative dose and relative potencies, which found the most effective in the reduction of POAF, seemed essential. ${ }^{26}$ (2) Except for the transient predisposing mechanisms of POAF, sympathetic activation seems to be more relevant than inflammation and oxidative stress. Withdrawal from and treatment with amiodarone or betablockers have been shown to largely affect POAF incidence, whereas reducing oxidative stress or inflammation was less effective with corticosteroid. (3) All corticosteroid drugs can cause sodium retention, resulting in dose-related fluid retention. The fluid retention directly affected left atrial wall tension, which has been shown to largely affect POAF incidence.

The PEG-based hydrogel used in the present study displays both biodegradability and immediate tissue adherence during in situ forming, allowing more localized drug delivery. Moreover, the clinical safety of epicardial application of this hydrogel has been demonstrated. ${ }^{27,28}$ Amiodarone/triamcinolone loading did not affect hydrogel tolerability in the current study. Amiodarone extravasation is not uncommon with peripheral vein infusions and can potentially cause serious injury and permanent harm, such as tissue necrosis. Previous animal studies provided in vivo evidence that topical amiodarone application has no direct toxic impact on atrial myocardium, but little is known about the structure and functional impairment of the atrial myocardium after epicardial amiodarone application in humans. In the current study, there are no significant changes on the positive value of troponin $\mathrm{T}$ release between the study groups and the control group, and there does not appear to be a higher incidence of atrial tachyarrhythmia in the study group. This compelling clinical evidence demonstrated that the epicardial amiodarone/triamcinolone application carries an extremely low risk of direct tissue injury.

\section{Study Limitations}

First, we did not evaluate the dose effects of the sustained release of amiodarone/triamcinolone from the adhesive hydrogels and kinetics after its release. "Intracardiac movement" has been suggested ${ }^{10,24}$ as a possible mechanism for absorption of pericardial application of amiodarone/triamcinolone from that space into the heart tissues. We speculate that a similar mechanism plays an important role in elevating the tissue concentration of amiodarone/triamcinolone in the atrium, although the precise mechanism was not investigated in our study. Second, only 1 dose of amiodarone hydrochloride powder or $1 \mathrm{~mL}$ triamcinolone suspension and 1 type of hydrogels were used to prepare drug-loaded hydrogels in this study. Variation of hydrogel formulations by changing polymer chemistry, altering drug dose, or adding epicardial steroid application may feature different drug-release characteristics. Third, the study design was selected on the basis of pilot experiments that demonstrated the surgical sealant did not contribute to the antiarrhythmic or anti-inflammatory effects. There is no amiodarone or triamcinolone in the hydrogel applied as placebo for the control group, Last, it is more interesting to investigate continuously biatrial electrophysiologic parameters perioperatively and measure atrial effective refractory periods and conduction times. 


\section{CONCLUSIONS}

Epicardial application of amiodarone-releasing adhesive hydrogel is a less-invasive, well-tolerated, quick to accomplish, and effective therapeutic option for preventing POAF with a minimal risk of extracardiac adverse side effects. However, there was no clinical evidence that epicardial corticosteroid prevented POAF.

\section{Conflict of Interest Statement}

Authors have nothing to disclose with regard to commercial support.

\section{References}

1. Almassi GH, Schowalter T, Nicolosi AC, Aggarwal A, Moritz TE, Henderson WG, et al. Atrial fibrillation after cardiac surgery a major morbid event? Ann Surg. 1997;226:501-13.

2. Villareal RP, Hariharan R, Liu BC, Kar B, Lee VV, Elayda M, et al. Postoperative atrial fibrillation and mortality after coronary artery bypass surgery. J Am Coll Cardiol. 2004;43:742-8.

3. Vassallo P, Trohman RG. Prescribing amiodarone: an evidence-based review of clinical indications. JAMA. 2007;298:1312-22.

4. Camm AJ. Safety considerations in the pharmacological management of atrial fibrillation. Int J Cardiol. 2008;127:299-306.

5. Aviles RJ, Martin DO, Apperson-Hansen C, Houghtaling PL, Rautaharju P, Kronmal RA, et al. Inflammation as a risk factor for atrial fibrillation. Circulation. 2003;108:3006-10.

6. Goldstein RN, Ryu K, Khrestian C, van Wagoner DR, Waldo AL. Prednisone prevents inducible atrial flutter in the canine sterile pericarditis model. J Cardiovasc Electrophysiol. 2008;19:74-81.

7. Tselentakis EV, Woodford E, Chandy J, Gaudette GR, Saltman AE. Inflammation effects on the electrical properties of atrial tissue and inducibility of postoperative atrial fibrillation. J Surg Res. 2006;135:68-75.

8. Halonen J, Halonen P, Jarvinen O, Taskinen P, Auvinen T, Tarkka M, et al. Corticosteroids for the prevention of atrial fibrillation after cardiac surgery: a randomized controlled trial. JAMA. 2007;297:1562-7.

9. Ayers GM, Rho TH, Ben-David J, Besch HR, Zipes DP. Amiodarone instilled into the canine pericardial sac migrates transmurally to produce electrophysiologic effects and suppress atrial fibrillation. J Cardiovasc Electrophysiol. 1996; 7:713-21

10. Darsinos JT, Karli JN, Samouilidou EC, Krumbholz B, Pistevos AC, Levis GM. Distribution of amiodarone in heart tissues following intrapericardial administration. Int J Clin Pharmacol Ther. 1999;37:301-6.

11. Takeda T, Shimamoto T, Marui A, Saito N, Uehara K, Minakata K, et al. Topical application of a biodegradable disc with amiodarone for atrial fibrillation. Ann Thorac Surg. 2011:91:734-9.

12. Bolderman RW, Hermans JB, Rademakers LM, De Jong MM, Bruin P, Dias AA, et al. Epicardial application of an amiodarone-releasing hydrogel to suppress atrial tachyarrhythmias. Int J Cardiol. 2011;149:341-6.
13. Konertz WF, Kostelka M, Mohr FW, Hetzer R, Hübler M, Ritter J, et al. Reducing the incidence and severity of pericardial adhesions with a sprayable polymeric matrix. Ann Thorac Surg. 2003;76:1270-4.

14. Zhibo X, Miaobo Z. Effect of sustained-release lidocaine on reduction of pain af ter subpectoral breast augmentation. Aesthet Surg J. 2009;29:32-4.

15. Miura S, Mii Y, Miyauchi Y, Ohgushi H, Morishita T, Hohnoki K, et al. Efficacy of slow-releasing anticancer drug delivery systems on transplantable osteosarcomas in rats. Jpn J Clin Oncol. 1995;25:61-71.

16. Auer J, Weber T, Berent R, Ng CK, Lamm B, Eber B. Postoperative atrial fibrillation independently predicts prolongation of hospital stay after cardiac surgery. J Cardiovasc Surg. 2005;46:583-8.

17. Kaireviciute D, Aidietis A, Lip GY. Atrial fibrillation following cardiac surgery: clinical features and preventative strategies. Eur Heart J. 2009:30:410-25.

18. Echahidi N, Pibarot P, O'Hara G, Mathieu P. Mechanisms, prevention, and treatment of atrial fibrillation after cardiac surgery. J Am Coll Cardiol. 2008;51: 793-801.

19. Bolderman RW, Hermans JJ, Rademakers LM, Jansen TS, Verheule S, Van der Veen FH, et al. Intrapericardial delivery of amiodarone and sotalol: atrial transmural drug distribution and electrophysiological effects. J Cardiovasc Pharmacol. 2009;54:355-63.

20. Hendrikx M, Mees U, Hill AC, Egbert B, Coker GT, Estridge TD. Evaluation of a novel synthetic sealant for inhibition of cardiac adhesions and clinical experience in cardiac surgery procedures. Heart Surg Forum. 2001;4:204-10.

21. Labhasetwar V, Underwood T, Heil RW Jr, Gallagher M, Langberg J, Levy RJ Epicardial administration of ibutilide from polyurethane matrices: effects on defibrillation threshold and electrophysiologic parameters. J Cardiovasc Pharmacol. 1994;24:826-40.

22. Kolettis TM, Kazakos N, Katsouras CS, Niokou D, Pappa L, Koulouras V, et al Intrapericardial drug delivery: pharmacologic properties and long-term safety in swine. Int J Cardiol. 2005;99:415-21.

23. Prasongsukarn K, Abel JG, Jamieson E, Cheung A, Russell JA, Walley KR. The effect of steroids on the occurrence of postoperative atrial fibrillation after coronary artery bypass grafting surgery: a prospective randomized trial. J Thorac Cardiovasc Surg. 2005;130:93-8.

24. Bruins P, te Velthuis H, Yazdanbakhsh AP, Jansen PG, van Hardevelt FW, de Beaumont EM, et al. Activation of the complement system during and after cardiopulmonary bypass surgery: postsurgery activation involves C-reactive protein and is associated with postoperative arrhythmia. Circulation. 1997;96:3542-8.

25. Yoo D, Vinten-Johansen J, Schmarkey SL, Whalen SP, Bone CC, Katzmark SL, et al. Adhesive epicardial corticosteroids prevent postoperative atrial fibrillation. Circ Arrhythm Electrophysiol. 2010;3:505-10.

26. Liu C, Wang J, Yiu D, Liu K. The efficacy of glucocorticoids for the prevention of atrial fibrillation, or length of intensive care unit or hospital stay after cardiac surgery: a meta-analysis. Cardiovasc Ther. 2014;32:89-96.

27. Vereckei A, Gorski JC, Ujhelyi M, Mehra R, Zipes DP. Intrapericardial ibutilide administration fails to terminate pacing-induced sustained atrial fibrillation in dogs. Cardiovasc Drugs Ther. 2004;18:269-77.

28. Wallace DG, Cruise GM, Rhee WM, Schroeder JA, Prior JJ, Ju J, et al. A tissue sealant based on reactive multifunctional polyethylene glycol. J Biomed Mater Res. 2001;58:545-55.

Key Words: postoperative atrial fibrillation, amiodarone, hydrogel 